\title{
CCD MOSAIC DEVELOPMENT FOR LARGE OPTICAL TELESCOPES
}

\author{
G. A. Luppino, M. R. Metzger \\ Institute for Astronomy, University of Hawaii \\ and S. Miyazaki \\ Institute for Astronomy, University of Hawaii and \\ Subaru Telescope Project, National Astr. Obs. of Japan
}

\begin{abstract}
We outline the recent developments in CCD imager technology aimed at producing the very large format $(8192 \times 8192$ pixels and larger) detector mosaics required for existing 4-m class and new 8 to $10-\mathrm{m}$ class telescopes. The key technology areas include buttable array design and buttable element packaging, and optimization schemes for QE and readout time. As an example, we highlight the University of Hawaii effort to develop an $8192 \times 8192$ $15 \mu \mathrm{m}$ pixel CCD mosaic.
\end{abstract}

\section{SCIENCE WITH LARGE CCD MOSAICS}

The need for larger and larger focal plane detectors has steadily increased due in part to two main technical advances: a) the steady improvement of image quality at observatories worldwide, and especially on Mauna Kea, and b) the demand for physically larger focal planes required for the next-generation 8 to 10-m telescope instruments. These are discussed in Sections 1.1 and 1.2 below.

\subsection{Imaging}

In recent years there has been a resurgence in "purely imaging" projects. For a time it was thought that direct imaging on large telescopes would give way to spectroscopy. This has not been the case. In fact a number of key scientific projects use both small (1 to 2-m) and large telescopes (4-m class) exclusively for imaging. Some current examples of such projects include: a) the surface brightness fluctuation (SBF) method for measuring distances to early-type galaxies out to $4000-5000 \mathrm{~km} \mathrm{~s}^{-1}$ (Tonry and Schneider 1988, Jacoby et al. 1992), b) the study of the dark matter distribution in clusters of galaxies from observations of gravitationally lensed arcs and arclets (Tyson et al. 1990, Kaiser and Squires 1993, Luppino et al. 1993), and, c) the search for baryonic dark matter (MACHOs) in our galactic halo from observations of gravitational microlensing of stars in the LMC (Stubbs et al. 1993, Griest 1991, also see Cook 1995 in these Proceedings). There are numerous additional scientific projects in galactic structure and solar system studies that, like the above, share a common need for large-format, high-QE detectors with high spatial resolution. By "large-format" we mean a detector with a field of view of exceeding $20^{\prime}$. By "high spatial resolution" we mean a detector that can adequately sample the 
seeing, which, for the best sites, implies a pixel size of order 0.2. In general, the science falls into two main categories: a) surveys, where we require observations of large numbers of objects or where we search large fields for objects with intrinsically low space density, and b) observations of intrinsically large objects.

\subsection{Detectors for Large Spectrographs}

Since the physical size of a focal plane scales with the size of the optical system, we can naturally expect that the focal planes required for the 8 to $10-\mathrm{m}$ telescope instruments will be quite large. The spectroscopic instruments planned for the Keck I and Keck II 10-m telescopes, the Subaru 8.2-m Japanese National Large Telescope (JNLT), and the Gemini 8-m telescopes will require detectors of order $100 \mathrm{~mm}$ format and larger. Moreover, for imaging applications it is often more cost effective and technically conservative to tile the large telescope focal planes with CCDs and bin pixels on-chip to achieve the desired pixel scale than to build extremely elaborate reimaging optics that may be more expensive than the CCDs, and may compromise the performance of the system. Most of the low resolution spectrograph designs call for at least a $4096 \times 4096$ CCD with 12 - $15 \mu \mathrm{m}$ pixels. Some of more ambitious instruments as well as the high resolution, cross-dispersed spectrographs and large imagers demand even larger formats. For example, the Keck DEIMOS spectrograph under development at UCSC requires two $8 \mathrm{k}$ x $8 \mathrm{k}$ (15 $\mu \mathrm{m}$ pixel) mosaics that are thinned, AR-coated, and mechanically flat and co-planar to within \pm five $\mu \mathrm{m}$ (see Stover et al. 1995 in these Proceedings).

\section{THE FIRST-GENERATION CCD MOSAICS: THE $4096^{2}$ DESIGNS}

A number of groups have developed or are developing CCD mosaics with $4096 \times 4096$ pixels. While there are some notable exceptions (Tyson et al. 1992, Sekiguchi et al. 1992), the majority of these mosaic designs are based on a family of two- and three-edge-buttable $2048 \mathrm{x}$ 2048 imagers designed by John Geary (e.g. see Geary et al. 1991) and fabricated at Loral Fairchild. The first of these close-packed $4096^{2}$ mosaics were successfully employed by the MACHO collaboration in their search for baryonic dark matter through observations of microlensing of stars from the LMC (Cook 1995, in these Proceedings). Similar mosaic prototypes have also been fabricated by the University of Hawaii (Luppino et al. 1992) and by NOAO (the "mini-mosaic", Boroson et al. 1994). An observer instrument for the CFHT, MOCAM (Cuillandre et al. 1995, in these Proceedings), will see first light this Fall. Many other mosaics and mosaic cameras are in the design or fabrication phase (see the list in Luppino, et al. 1994). Fig. 1 shows an example of a typical first-generation $4096^{2}$ mosaic. This drawing shows a two by two array of two-edge-buttable CCDs mounted on modified kovar packages.

\section{STRATEGIES FOR LARGER MOSAICS}

While a $4096^{2}$ mosaic is certainly useful for a variety of observational projects, for many projects such a mosaic offers only marginal improvement over what one can accomplish with a single thinned Tek 2048 CCD. A $4096^{2}$ mosaic with $15 \mu \mathrm{m}$ pixels is only slightly larger (60 $\mathrm{mm}$ ) than the monolothic Tek 2048 with $24 \mu \mathrm{m}$ pixels $(49 \mathrm{~mm})$. In situations where the smaller pixels are of no advantage (e.g. direct imaging on the UH 2.2-m telescope), the thinned Tek $2048 \mathrm{CCD}$ is superior to an unthinned $4096^{2}$ mosaic. Thus, the UH $4096^{2}$ mosaic was a stepping stone to the larger mosaics that we require for wide field imaging cameras and large format spectrograph readouts. 


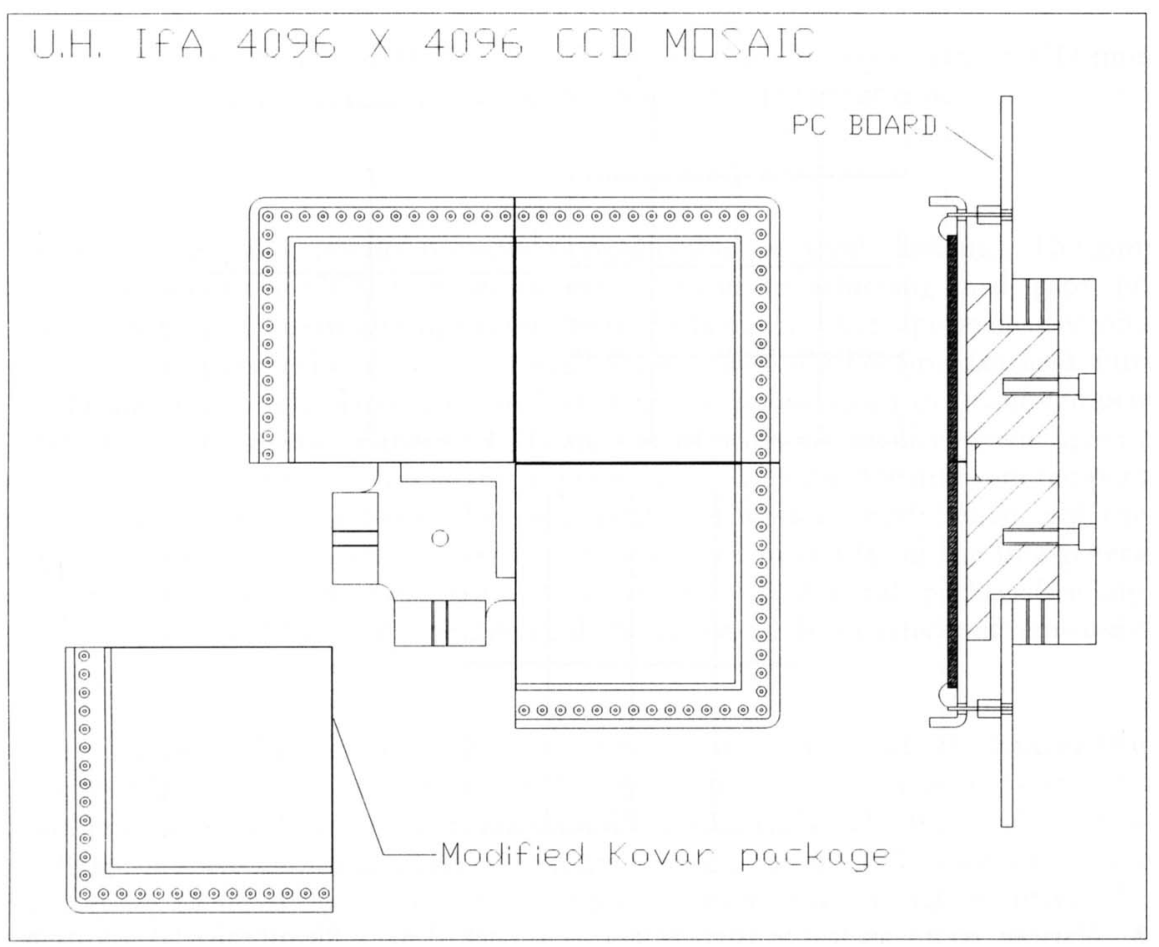

Fig. 1. The 4096 x 4096 CCD mosaic prototype design built by the University of Hawaii in 1992. This is the same design employed in MOCAM for the CFHT (see Cuillandre et al. 1995, in these Proceedings).

What approach can we take to move to the next level in mosaic design? Consider that our goal is to construct a detector with $8192 \times 819215 \mu \mathrm{m}$ pixels. When designing such a large CCD mosaic, a number of approaches can be taken, trading off mosaic tile size, gap size, number of amplifiers, readout time, etc. First, we address gap size. In some applications, especially wide field imaging, relatively large gaps are not considered a problem since deep images are often obtained by taking many unregistered, short exposures, thus "filling" the gaps while allowing removal of cosmic rays and improved flatfielding. For spectrograph focal planes, however, gaps can be a serious nuisance, especially for multiobject and cross-dispersed instruments. Therefore, for the remainder of this paper we consider only close-packed mosaics.

Next we consider the optimal CCD tile size. It is now routine to fabricate $2048 \times 204815$ $\mu \mathrm{m}$ pixel CCDs with excellent yield. Four devices can fit on a $100 \mathrm{~mm}$ wafer, and a foundry run will produce 80 devices, often with more than half the devices electrically functioning and a sizeable fraction of scientific quality. Furthermore, a variety of designs with two and 


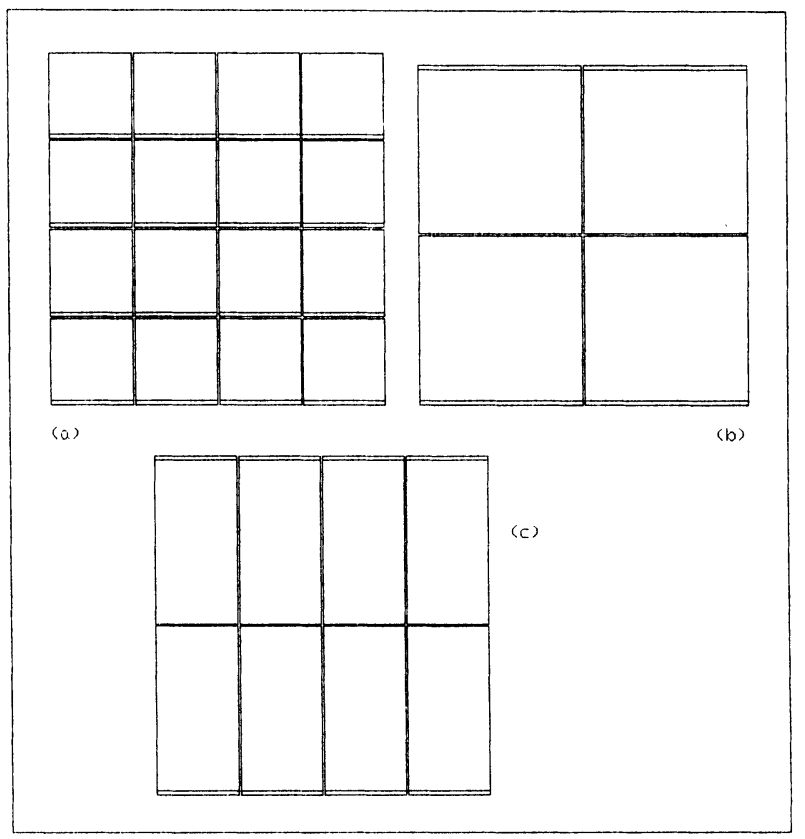

Fig. 2. Various strategies for constructing a close-packed $8 \mathrm{k} \mathrm{x} 8 \mathrm{k}$ mosaic: (a) a four by four array of three-edge-buttable $2 \mathrm{k} \times 2 \mathrm{k}$ CCDs, (b) a two by two array of three (or two) edge buttable $4 \mathrm{k} \times 4 \mathrm{k}$ CCDs, and (c) a four by two array of $2 \mathrm{k} \mathrm{x} 4 \mathrm{k}$ three-edge-buttable CCDs.

three-edge buttability and standard quad amp readout exist for these 2048 devices (Geary et al. 1991). One could assemble an $8192 \times 8192$ mosaic from 16 of these $2048^{2}$ imagers arranged in a four by four array. Unfortunately, some or all of the gaps would exceed one $\mathrm{mm}$, and unless four-side-buttable packaging were developed, the gaps could be considerable ( $\geq 1 \mathrm{~cm})$. A mosaic with $16 \mathrm{CCD}$ elements would also be electrically and mechanically complex, requiring numerous readouts, and making alignment and flatness difficult to achieve (see Fig. 2a).

Considering the high yield on the 2048 devices, we could attempt to build a 4096 x 4096 two-edge or three-edge-buttable CCD with $15 \mu \mathrm{m}$ pixels (Fig. 2b). This device would fill the $100 \mathrm{~mm}$ wafer and the yield would be considerably lower than for the $2048 \mathrm{CCDs}$. But the resulting $8192 \times 8192$ could be assembled from a simple two by two array of these devices in a scaled-up version of the first generation mosaics described in the previous section. Loral Fairchild has attempted on several occasions to build such a device. They have not been successful, however, in producing any scientific quality imagers. Rather than pursue this strategy, one could adopt an intermediate solution and choose a design that is midway between the buildable $2 \mathrm{k}^{2}$ imagers and the ambitious $4 \mathrm{k}^{2}$ imagers: a 2048 x 4096 three-buttable design with $15 \mu \mathrm{m}$ pixels. Two of these devices would fit onto a $100 \mathrm{~mm}$ wafer, thus significantly increasing the expected yield. A close-packed $8 \mathrm{k}^{2}$ mosaic can be built from a four by two array of such imagers. 


\section{CRITICAL DESIGN ISSUES}

In this Section, we touch on three key technology areas relating to large CCD mosaics: a) mosaic packaging, b) thinning and QE optimization, and c) readout time.

\subsection{Mosaic Packaging}

Packaging large CCD mosaics presents a considerable technical challenge. The simplest approach is to mount the CCD tiles on a common substrate, achieving small gaps, precise alignment and flatness in the mounting process. Such a scheme, however, permanently joins the CCD dies and precludes replacement of a mosaic element should it become damaged. Furthermore, CCD dies are usually selected for inclusion in the mosaic based on room-temperature wafer-probe tests, and many of these CCDs are not of scientific quality when operated at cryogenic temperatures. Thus, a mosaic that is permanently assembled from room-temperatureselected dies may contain a number of elements that do not work properly when cold, thereby destroying the entire mosaic. The preferred technique used in nearly all the first-generation, close-packed mosaics was to employ a custom-designed, two-edge-buttable package (see Luppino et al. 1992, Luppino and Miller 1992, Stubbs et al. 1993) that can be inserted and removed easily from a mosaic.

Although a three-edge-buttable package is somewhat more technically challenging than the two-edge-buttable designs, we can still adopt the same strategy for the larger mosaics as for the earlier mosaics; individual mosaic elements should be replaceable. The technical issues in the package design involve choice of materials, method of fabrication, and method for achieving electrical contact. Material choices include ceramics (e.g. aluminum nitride) and metals (Kovar, Invar, molybdenum). Aluminum nitride is an excellent choice for a package material, but it must be worked by the manufacturer. It is also non-conductive which precludes electrical contact to the CCD substrate if that is necessary. Kovar packages were employed almost exclusively in the first generation mosaics. It turns out, however, that Kovar is a poor thermal match to silicon, but was used because it is a common, commercially available microelectronics package material (Kovar's popularity in the microelectronic industry results not from any match to silicon, but because Kovar has a coefficient of thermal expansion precisely matched to the glass used to mount and insulate the pins in these packages, allowing such packages to be hermetically sealed and used over a wide temperature range). If we are to match the coefficient of thermal expansion (CTE) of silicon, we must choose an appropriate metal, such as Invar 36 (Szentgyorgyi 1993) or molybdenum. While Invar has the better CTE match, molybdenum is a far better thermal conductor (by nearly an order of magnitude), and may prove to be the better material overall.

In either case, the material can be machined to the required close tolerances using conventional (grinding) or EDM machining. An important consideration for the package design is maintaining focal plane flatness. If the large mosaics are to be used in fast optical beams (e.g. prime focus cameras of spectrographs) then the deviation from flatness cannot exceed of order a pixel size to maintain focus across the array. This specification places severe constraints on the mechanical package specifications which must be held flat to of order $\pm 10 \mu \mathrm{m}$. 


\subsection{Thinning and QE Optimization}

It is well understood that to achieve the highest possible quantum efficiency from these CCD mosaics, the individual CCDs must be thinned, back illuminated and anti-reflection (AR) coated. Thinning exposes the bare silicon surface that can be anti-reflection coated to allow the incident photons to enter the device. Backside treatments are then necessary to ensure that the photoelectrons are actually detected and not caught in surface traps generated by the thinning process. While thinning conventional CCDs is hard enough, thinning edge-buttable CCDs is harder still. It is important that thinning techniques are developed that thin the entire device and do not leave a "lip" near the buttable edges. This may require full wafer scale thinning where the entire CCD wafer is thinned and processed before dicing the individual CCD elements.

An enormous amount of effort has been expended developing the technology for thinning CCDs. Much of the present day techniques have been pioneered by groups like Lesser's at the UA Steward Observatory, and we refer readers to the many papers in this area (e.g. see Lesser 1994 and references therein). While CCD quantum efficiency in the blue and mid visible depends on the AR-coating efficiency and the properties of the thinned backside interface, the $\mathrm{QE}$ redward of $\sim 800 \mathrm{~nm}$ depends primarily on the AR-coating and the on the thickness of the thinned silicon membrane. Although the CCD has no response at wavelengths longer than 1100 $\mathrm{nm}$, there is a scientifically critical area between $900 \mathrm{~nm}$ and $1100 \mathrm{~nm}$ where boosted CCD response will have enormous scientific gains. The one sure way to increase CCD response in this area is simply to build thicker devices (note, however, that we still need thinned, back illuminated devices so we can $\mathrm{AR}$ coat the backside for the highest sensitivity). Since we presently thin devices to around $15 \mu \mathrm{m}$, would it be possible to build devices with a $30 \mu \mathrm{m}$ thick epitaxial layer (EPI) and thin to $\sim 30 \mu \mathrm{m}$ ? This is certainly possible, and will result in higher red $\mathrm{QE}$, but such a device built on conventional $10-50 \Omega \mathrm{cm}$ silicon will experience charge spreading since this thicker EPI will only be depleted a few $\mu \mathrm{m}$ deep, and charge generated in the "field free" region below the depletion region will diffuse laterally and contaminate neighboring pixels, resulting in a loss of image sharpness. On the other hand, we can build thicker devices and avoid the image sharpness tradeoff by building the CCDs on high resistivity silicon. The depth of the depletion region depends both on the gate voltage and the resistivity of the silicon. For a fixed gate voltage depletion depth is proportional to the square root of the resistivity. Thus to fully deplete a $30 \mu \mathrm{m}$ membrane, we need to build CCDs on material with approximately ten times the resistivity of conventional CCDs (i.e. $1000-5000$ $\Omega \mathrm{cm}$ silicon). Such "deep depletion" CCDs have been pioneered by the X-ray astronomy community where high energy ( 5 to $10 \mathrm{keV}$ ) X-ray response is analogous NIR response (see Burke et al. 1991). An added advantage of thicker CCDs is the reduction of interference fringing.

\subsection{Readout Time}

As we attempt to build larger CCD mosaics, is crucial that we address the problem of readout time. For applications where we are detector noise limited, such as high dispersion spectroscopy or narrow band imaging, the lowest redout noise possible is essential. The lowest noise levels achieved with commercially-available CCDs are around $2.5-3 \mathrm{e}^{-}$. These noise levels are only possible, however, by reading out the CCD in "slow scan" mode with a pixel rate in the range of $20-50 \mathrm{kHz}$ (kpixels/sec). For the large arrays we intend to build, however, such 
a slow readout speed is a severe limitation, since it leads to objectionably long readout times; nearly seven minutes for a single $2 \mathrm{k}$ x $2 \mathrm{k} \mathrm{CCD}$ at $20 \mathrm{kHz}$. Long readout times present a serious efficiency problem in applications where one is taking many short exposures, such as broad band imaging. Most deep imaging observations are obtained using the "shift and stare" technique where one takes many short exposures while shifting the telescope slightly between the exposures, thus allowing one to build up a "flatfield" using the disregistered images. Taking many short exposures also helps with the removal of cosmic ray hits in the CCD images. When this technique is used on present-day 4-m class telescopes, the individual exposures are often limited to five to ten minutes each. On 10-m telescopes, it would be even better to use only one to two minute exposures. The limitation on the exposure time is dependent on the readout noise level of the CCD and the brightness of the night sky (we integrate until the sky photon shot noise dominates the detector readout noise).

Clearly, if we are taking many exposures, and reading out the CCD between those exposures, our observing efficiency is strongly affected by the CCD readout time. If the readout time is of order five minutes and our exposures are of order five minutes, then half the night is wasted reading out the CCD instead of collecting data from astronomical objects. One approach taken to offset this problem is to build CCDs with multiple output amplifiers, thus reducing the readout time by reading through two or four amplifiers in parallel. While this approach certainly solves the problem, it introduces other drawbacks that are only now being realized in recently constructed systems using multiple on-chip amplifiers. First, in a multiple amplifier CCD or mosaic, each separate amplifier must be calibrated. And that calibration must remain stable over time. The standard observational approach for calibrating one's data is to observe standard stars through the exact same telescope optics and atmosphere as the data were taken. But with multiple amplifier configurations, one needs to place standard stars on all regions of the CCDs to calibrate all the amplifiers. Trusting that one can calibrate with just one amplifier using previously determined relative amplifier gains is a risky business, and is not adequate for precise photometry. An additional problem is amplifier to amplifier crosstalk that can appear in multiple on-chip amplifier configurations. This can be a difficult problem in astronomical observations where objects of enormous brightness difference can be present on single CCD frames. We therefore argue that minimizing the number of amplifiers is desirable. But then how do we decrease the readout time? Clearly the best solution is to increase the readout speed, without increasing the readout noise beyond acceptable levels.

This goal is possible with present day technology. Manufacturers, however, must integrate the appropriate amplifiers into their designs. As an example of what can be done now, we show in Fig. 3 the readout noise as a function of readout speed for a MIT Lincoln Labs (MITLL) CCID-10 $1024 \times 2048$ CCD measured in the UH IFA CCD Lab. Our CCD controller electronics uses a dual slope integrator implementation of a correlated double sampler circuit, and plotted on the $\mathrm{x}$-axis of Fig. 3 is the dual slope integrator integration time. The conventional dual slope integrator integrates "up" on the reset level and "down" on the signal level, thus the minimum pixel time is twice the dual slope integrator integration time. Certain values for the corresponding pixel frequency (in kps or kpixels/sec) are also shown on the graph. This MITLL amplifier achieves a noise floor of $1.7 \mathrm{e}^{-} \mathrm{rms}$ per pixel at a conventional slow-scan rate of $50 \mathrm{kHz}$, but has a readout noise below five $\mathrm{e}^{-}$at a speed as fast as $500 \mathrm{kHz}$. The readout time for a $2 \mathrm{k} \mathrm{x} 4 \mathrm{k} \mathrm{CCD}$ at $500 \mathrm{kpixels} / \mathrm{sec}$ is only 16 seconds! Clearly, amplifiers of this type should be incorporated into CCD designs that are intended for large format mosaics. 


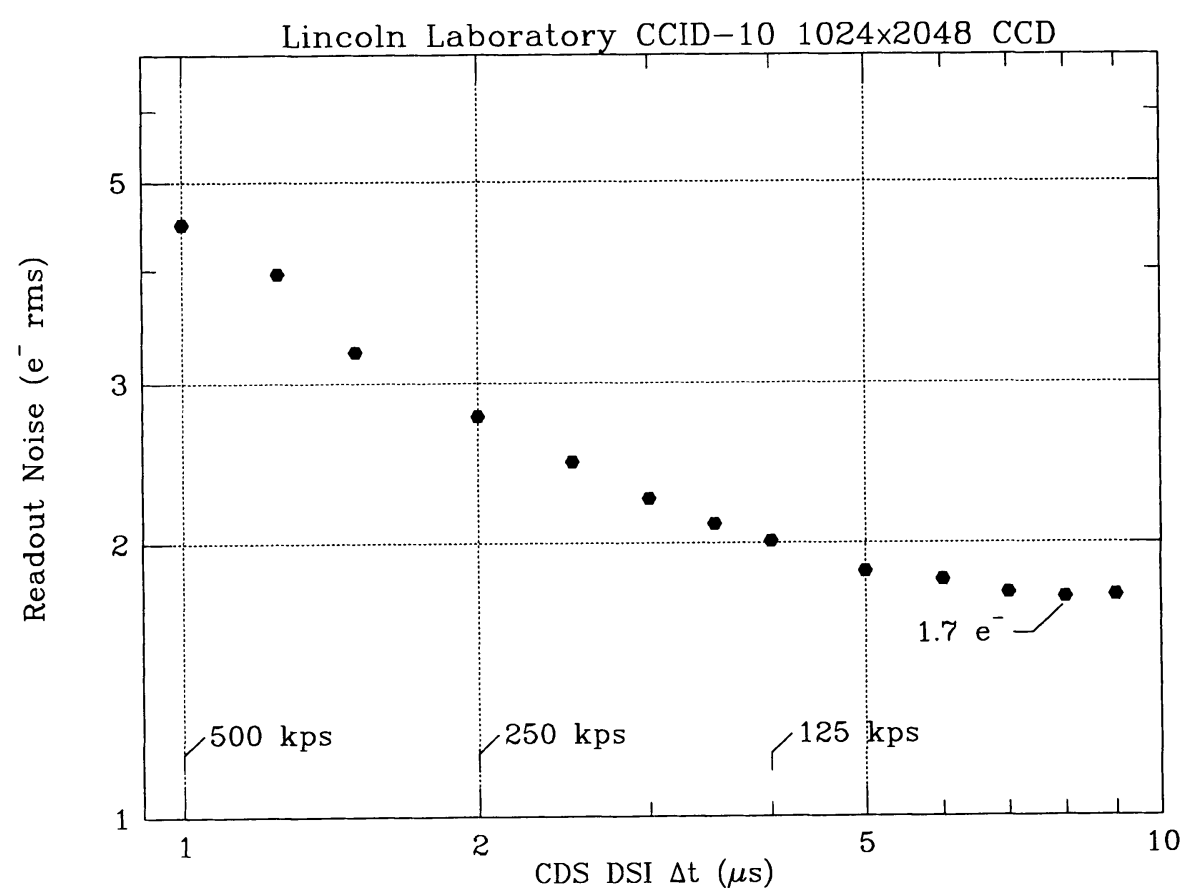

Fig. 3 This plot illustrates that low noise, high speed operation is possible with state-of-the-art CCD amplifier designs. The data were taken at the UH IFA CCD Lab with a MITLL CCID-10 CCD.

\section{THE UH $8192 \times 8192$ CCD MOSAIC DESIGN}

The University of Hawaii, Institute for Astronomy is presently building an $8 \mathrm{k} \mathrm{x} 8 \mathrm{k} \mathrm{CCD}$ mosaic camera for use on the various telescopes on Mauna Kea. In this Section, we will describe this camera as an example of a state-of-the-art astronomical CCD mosaic.

The UH $8 \mathrm{k}$ mosaic camera is based on a $2 \mathrm{k} \mathrm{x} 4 \mathrm{k}$ three-edge-buttable CCD fabricated at Loral Fairchild (see Luppino, Bredthauer and Geary 1994). The device is designed to be three-edge-buttable with the single serial register running along a short (2048) edge. All of the bond pads are also confined to this edge. We can construct an 8192 × 8192 mosaic from a two by four array of these devices. The single serial register has a standard floating diffusion LDD amplifier at each end, and is split to allow the CCD to be read out of either amplifier or out of both simultaneously (note this is the same device design being used by NOAO in their $8 \mathrm{~K}$ mosaic project; Boroson et al. 1994). A single $2 \mathrm{k} \mathrm{x} 4 \mathrm{k}$ mosaic element and its associated three-edge-buttable package is shown in Fig. 4. The CCD die is attached via electrically conductive sheet epoxy to a custom molybdenum package. Mounted adjacent to the CCD bonding pads is a small PC board containing a 25-pin micro-D connector. The CCD is wire bonded directly to the PC board. The CCD and package are designed so that the gaps in the resulting mosaic are less than one $\mathrm{mm}$. 

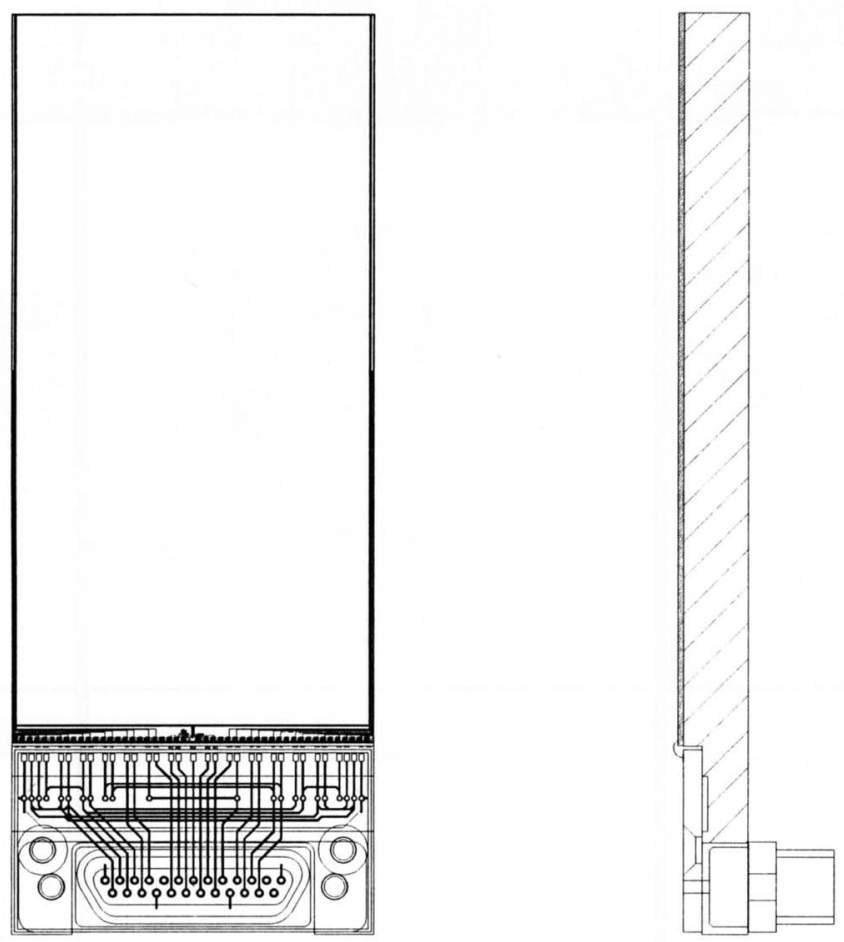

Fig. 4. The Loral $2 \mathrm{k}$ by $2 \mathrm{k}$ three-edge-buttable CCD and the buttable package design being developed at the UH IFA. The frontside illuminated CCD die is attached directly to a precision machined molybdenum carrier. A PC board containing a micro-D connector is also attached to the carrier and is wire bonded to the CCD.

The eight mosaic elements mount to a common base suspended from thermally insulated supports behind a $12 \mathrm{~mm}$ thick quartz window in a large LN2 cooled, vacuum cryostat. Each individual mosaic element will be precisely aligned using a microscope and precision alignment fixture. A scale drawing of the mosaic focal plane is shown in Fig. 5.

The first light instrument will be equipped with a large focal plane shutter and a two position filter slide for $150 \mathrm{~mm}$ square filters. Design and fabrication work is underway on these components. The lot run of CCDs at Loral Fairchild was reasonably successful, with the first 20-wafer lot (40 devices) produced 16 functional devices, 12 of which appear to be of scientific quality in room temperature wafer probe tests. We will shortly test these devices at cryogenic temperatures to select the eight best devices for inclusion in the mosaic. 


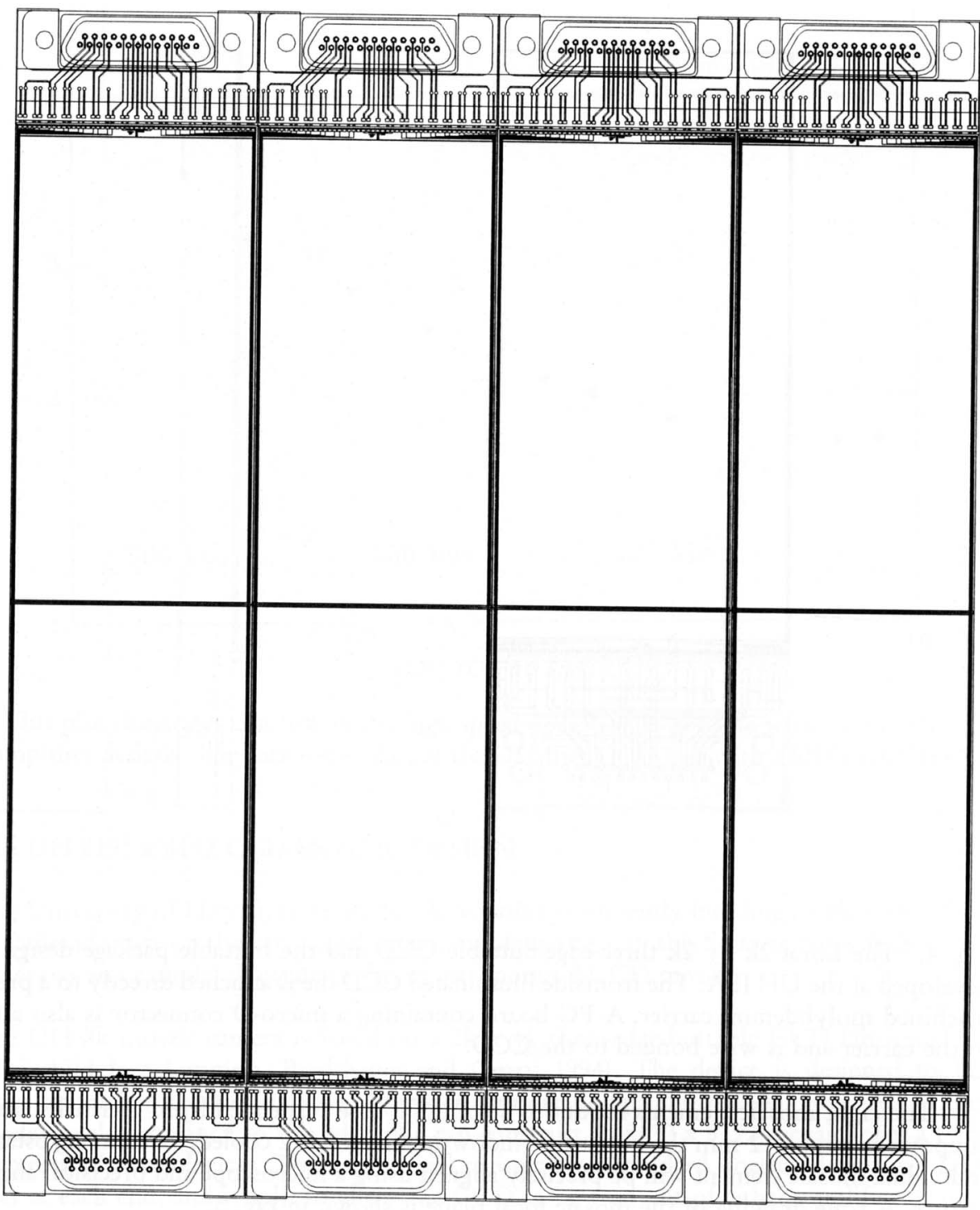

Fig. 5. The University of Hawaii's $8192 \times 8192$ CCD mosaic. The resulting active area is 122.9 $\mathrm{mm} \times 122.9 \mathrm{~mm}$. The gaps are less than $1 \mathrm{~mm}$. 


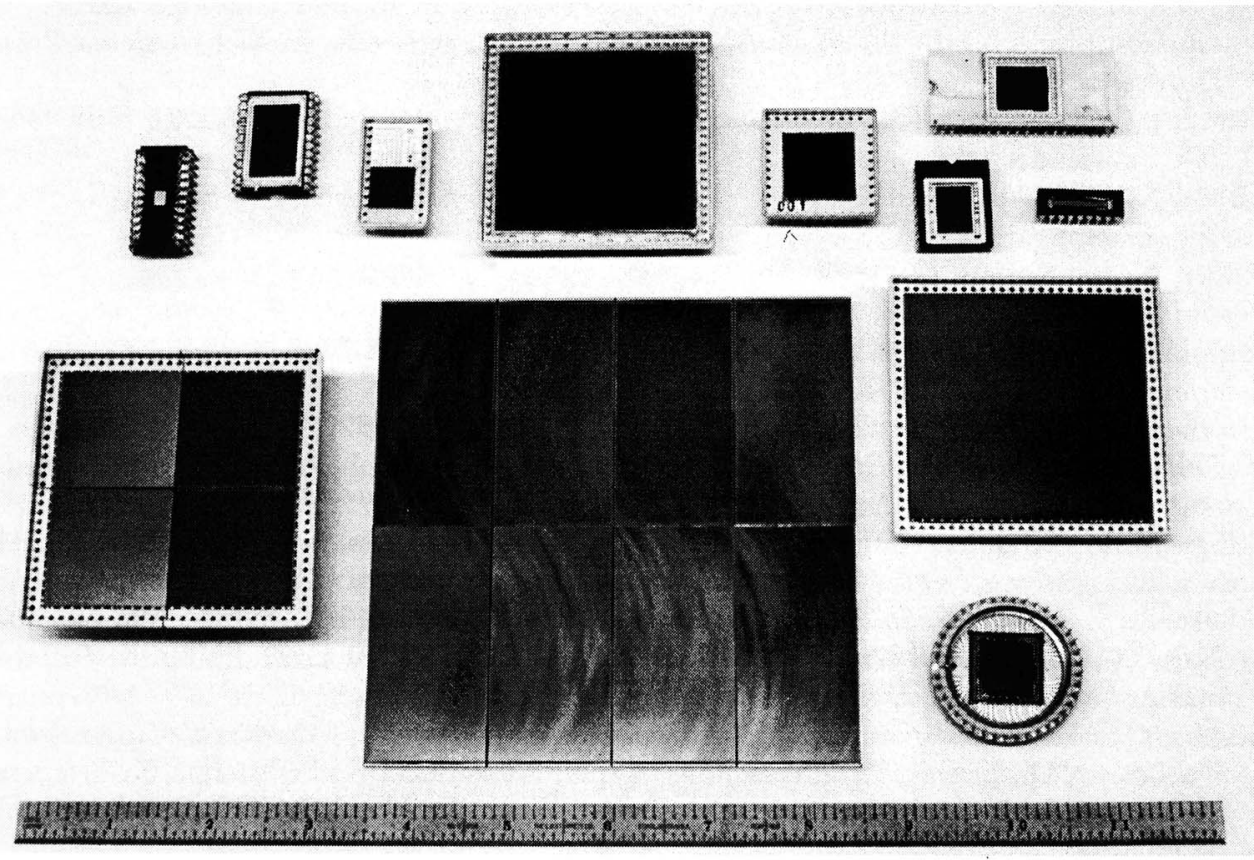

Fig. 6. A photograph of a non-functional $8 \mathrm{k} \times 8 \mathrm{k}$ mosaic formed from eight dead $2 \mathrm{k} \times 4 \mathrm{k}$ devices. This is shown for comparison to a $4096 \times 4096$ mosaic (at the left), a Tedtronix 2048 x 2048 CCD (at the top) and a Loral $4096^{2}$ monolithic (15 $\mu \mathrm{m}$ pixel) device (at the right. Various other smaller CCDs of historical significance are also shown (TI $800^{2} \mathrm{WF} / \mathrm{PC}$ CCD at lower right, Fairchild $100^{2}$ at upper left and to its right an RCA 512 × 320.

This $8 \mathrm{k}$ mosaic camera is a step toward future, improved versions large format detector focal planes. In its first incarnation, this camera will use frontside illuminated CCDs with a peak QE of only $\sim 40 \%$. Thinning is an option for future mosaics, as is deep depletion. We will explore the compromise between readout noise and readout speed, but we have not yet incorporated new on-chip amplifier designs that are optimized for low noise at higher speeds. Nevertheless, we intend to use this mosaic camera for several wide field imaging projects on the CFHT 3.6-m, the UH 2.2-m and the UH 0.6-m telescopes where the substantial increase in field of view will outweigh the compromises in other areas.

\section{REFERENCES}

Boroson, T., Reed, R., Wong, W.-Y. and Lesser, M. 1994 Proc. SPIE 2198, 877

Burke, B., Mountain, R., Harrison, D., Bautz, M., Doty, J., Ricker, G. and Daniels, P. 1991 IEEE Trans. Elec. Dev. 38, 1069

Cook, K. H. 1995 in IAU Symposium No. 167, New Developments in Array Technology and Applications, A. G. D. Philip, K. A. Janes and A. R. Upgren, eds., Kluwer Academic Press, Dordrecht, p. 285 
Cuillandre, J. C., Murrowinski, R., Crampton, D., Mellier, Y., Luppino, G. and Arsenault, R. 1995 in IAU Symposium No. 167, New Developments in Array Technology and Applications, A. G. D. Philip, K. A. Janes and A. R. Upgren, eds., Kluwer Academic Press, Dordrecht, p. 213

Geary, J. C., Luppino, G. A., Bredthauer, R., Hlivak, R. J. and Robinson, L. 1991 SPIE 1447, 264

Griest, K 1991 ApJ 366, 412

Jacoby, G. et al. 1992 PASP 104, 599

Kaiser, N. and Squires, G. 1993 ApJ 404, 441

Lesser, M. 1994 SPIE 2198, 782

Luppino, G. A., Bredthauer, R. and Geary, J. 1994 SPIE 2198, 810

Luppino, G. A. and Miller, K. 1992 PASP 104, 215

Luppino, G. A., Jim, K. T. C., Hlivak, R. J. and Yamada, H. 1992 SPIE 1656,414

Luppino, G. A., Gioia, I., Annis, J., Hammer, F. and Lefevre 1993 ApJ 416, 444

Sekiguchi, M., Iwashita, H., Doi, M., Kashikawa, N. and Okamura, S. 1992 PASP 104, 744

Stover, R. J., Brown, W. E., Gilmore, D. K. and Wei, M. 1994 SPIE 2198, 803

Stover, R. J., Brown, W. E., Gilmore, D. K. and Wei, M. 1995 in IAU Symposium No. 167, New Developments in Array Technology and Applications, A. G. D. Philip, K. A. Janes and A. R. Upgren, eds., Kluwer Academic Press, Dordrecht, p. 19

Stubbs, C., Marshall, S., Cook, K., Hills, R., Noonan, J., Akerlof, C., Alcock, C., Axelrod, T., Bennet, D., Dagley, K., Freeman, K., Griest, K., Park, H.-S., Perlmutter, S., Peterson, B., Quinn, P., Rodgers, A., Sosin, C. and Sutherland, W. (The MACHO Collaboration) 1993, SPIE 1900, 192

Szentgyorgyi, A. 1993 Harvard CfA/SAO Technical Memorandum

Tonry, J. and Schneider, D. 1988 AJ 96, 807

Tyson, J. A., Bernstein, G., Blouke, M. and Lee, R. 1992 SPIE 1656, 400

Tyson, J. A., Valdes, F. and Wenk, R. A. 1990 ApJ 349, L1

\section{DISCUSSION}

IWERT: I think it is worth mentioning that the bottleneck in large CCDs is (and probably will be) the thinning of those devices, although many small groups are working on it. We can certainly build giant mosaics of frontside illuminated CCDs, but which additional options besides the well known groups do you see for thinning in the future?

LUPPINO: There are vendors, most notably SITE, who claim they are developing thinned $2 \mathrm{k}$ by $2 \mathrm{k}$ CCDs. And of course, there is the Foundry Kesser route. But we don't have to wait for thinned devices to do good science.

TINBERGEN: To add a point to your list of science drivers: I think photometry will benefit greatly from over-sampling and slower "camera" beams.

LUPPINO: Yes I agree. I have a very "personally biased" list of science drivers. 